\title{
Influência da composição do gás de proteção nas tensões residuais de juntas de aço DP600 soldadas pelo processo GMAW
}

\author{
Influence of shielding gas composition on \\ the residual stresses of DP600 steel \\ joints welded by GMAW process
}

Thiago de Menezes Maia ${ }^{1}$, Hector Reynaldo Meneses Costa ${ }^{1}$, Maria Cindra Fonseca ${ }^{2}$,Tatiane de Campos Chuvas ${ }^{1}$

\begin{abstract}
${ }^{1}$ Programa de Pós Graduação em Engenharia Mecânica e Tecnologia dos Materiais, PPEMM, Centro Federal de Educação Tecnológica Celso Suckow da Fonseca, CEFET-RJ, Rio de Janeiro, Rio de Janeiro, Brasil.

${ }^{2}$ Departamento de Engenharia Mecânica, Programa Francisco Eduardo Mourão Saboya de Pós-Graduação em Engenharia Mecânica, PGMEC, Universidade Federal Fluminense - UFF, Niterói, Rio de Janeiro, Brasil.

e-mail: thiagommaia@gmail.com, hectorey@gmail.com, tatiane.chuvas@cefet-rj.br, mcindra@vm.uff
\end{abstract}

\section{RESUMO}

A indústria automobilística vem investindo em novos processos de fabricação que melhoram a produtividade sem perda da qualidade. Entretanto, a presença de tensões residuais, geradas em todos os processos de fabricação, constitui um dos grandes problemas encontrados na indústria metal mecânica, pois estas podem se somar às tensões de carregamento externo e causar falhas catastróficas em equipamentos e componentes. Sendo a soldagem um dos processos de fabricação mais utilizados atualmente, é necessário entender como os parâmetros de soldagem impactam na natureza e magnitude das tensões residuais geradas. Em alguns processos de soldagem são utilizados gases de proteção, que podem influenciar a condutividade do arco elétrico e, consequentemente, afetar o comportamento das tensões residuais nas juntas soldadas. Neste trabalho foi realizado um estudo experimental sobre a influência do percentual de $\mathrm{CO}_{2}$ dos gases de proteção com base em argônio, nas tensões residuais geradas em juntas de aço avançado de alta resistência DP600, soldadas pelo processo GMAW (amplamente utilizado na indústria automobilística). As tensões residuais foram medidas por difração de raios-X, pelo método do $\operatorname{sen}^{2} \psi$. A avaliação microestrutural foi realizada através de microscopia óptica. Os resultados mostraram que as tensões residuais têm natureza trativa, da ordem de $120 \mathrm{MPa}$, em todas amostras que utilizaram gás de proteção com menor percentual de $\mathrm{CO}_{2}$.

Palavras-chave: aço DP600, GMAW, tensões residuais, gases de proteção, difração de raios-X.

\begin{abstract}
The automotive industry has been investing in new manufacturing processes that improve productivity without loss of quality. However, the presence of residual stress (RS), generated in all manufacturing processes, is one of the major problems encountered in the metalworking industry, since these can add up to external loading stresses and cause catastrophic failures in equipment and components. Since welding is one of the most widely used manufacturing processes, it is necessary to know how the welding parameters impact on the nature and magnitude of the residual stresses generated. The shielding gases are used in some welding processes and can be influence the conductivity of the electric arc, which can be affected the residual stresses behavior in the welded joints. In this work an experimental study was carried out about the influence of the percentage of $\mathrm{CO}_{2}$ on the argon-based shielding gases in the residual stresses generated on joints of high strength steel DP600 welded by GMAW process (widely used in the automotive industry). The residual stresses were measured by X-ray diffraction by the $\sin ^{2} \psi$ method. An assessment of the microstructural morphology by optical microscope (OM) was made. The results showed that the residual stresses have a tensile nature, of the order of $120 \mathrm{MPa}$, in all samples, which used shielding gas with a lower percentage of $\mathrm{CO}_{2}$.
\end{abstract}

Keywords: DP600 steel, GMAW, residual stresses, shielding gases, X-ray diffraction. 


\section{INTRODUÇÃO}

Os aços DP (Dual - Phase) surgiram na década de 1970 e nos últimos 20 anos começaram a ser utilizados pela indústria automobilística, que vem incentivando o avanço metalúrgico em busca de materiais de alta resistência e alta conformabilidade, com o intuito de reduzir o peso dos automóveis e, consequentemente, o consumo de combustível e a emissão dos poluentes [1]. Os aços DP apresentam microestrutura composta basicamente por uma matriz ferrítica $(80$ a $85 \%)$ e ilhas de martensita $(10-15 \%)$. Esses aços apresentam alta conformabilidade e ductilidade devido à presença de uma matriz ferrítica, sendo a resistência mecânica relacionada à fase martensítica [2]. No processo de fabricação desses aços ocorre um aquecimento na zona intercrítica, propiciando a formação de núcleos de austenita na matriz ferrítica, seguido de um rápido resfriamento, ocorrendo a transformação de austenita para martensita [3,4]. Conforme a norma SAE J2745 [5], o aço DP600 deve possuir um limite de escoamento entre 340 - $440 \mathrm{MPa}$, resistência à tração mínima de $590 \mathrm{MPa}$ e alongamento na faixa de $21 \%$.

Os processos de soldagem têm sido amplamente empregados na indústria automobilística. Entretanto, a geração das tensões residuais, devido ao aquecimento em uma região localizada com mudança de fase na zona termicamente afetada (ZTA) e no metal de solda (MS), além de um resfriamento rápido e não uniforme na junta com gradientes térmicos entre a peça e o núcleo da junta, é um dos grandes problemas desse setor. Segundo WHITERS et al. [6], as tensões residuais (TR) são tensões existentes nos materiais, sem gradientes de temperatura e carregamentos externos. A natureza das tensões residuais originadas durante a soldagem pode ser devida ao aquecimento localizado, acompanhado por um resfriamento com elevado gradiente térmico que surge a partir da zona de solda. Assim, transformações de fase no metal de solda e na zona termicamente afetada influenciam diretamente a distribuição das tensões residuais e juntas soldadas [7]. Com isso, a vida útil do material quanto à resistência à fadiga pode ser influenciada pela natureza dessas tensões, uma vez que tensões residuais trativas são prejudiciais por facilitar a nucleação e propagação de trincas. Por outro lado, as tensões de natureza compressivas são benéficas por dificultar a nucleação e a propagação de trincas, reduzindo a tensão total na superfície da peça [8-9].

Em particular, o processo de soldagem GMAW (Gas Metal Arc Welding - Soldagem por arco elétrico com gás de proteção) é bastante utilizado nas indústrias em geral devido à alta produtividade, aplicação em uma ampla faixa de espessura e diferentes posições de soldagem, adaptabilidade para automatização ou mecanização do processo, além da alta taxa de deposição [10]. Evitar a contaminação do ar atmosférico na poça de fusão e manter a estabilidade do arco elétrico são as principais funções dos gases de proteção [11]. O argônio puro não é muito utilizado em soldagem de aços devido a alguns fatores, tais como baixa condutividade térmica e por não apresentar uma boa estabilidade no arco elétrico. Deste modo, adições de $\mathrm{CO}_{2}$ são usadas na soldagem de aços carbono e baixa liga, com o intuito de conseguir um bom equilíbrio na incidência de respingos e penetração e boa estabilidade de arco com maiores velocidades. Além disso, a junta apresenta maiores penetrações e menos porosidade quando se utiliza adições de dióxido de carbono no gás de proteção [12]. Contudo, o aumento da quantidade de $\mathrm{CO}_{2}$ na mistura pode causar um aumento considerável na corrente de transição e minimizar a condutividade do arco elétrico [13].

O objetivo desse trabalho é caracterizar, no âmbito das tensões residuais, a influência do $\mathrm{CO}_{2}$ dos gases de proteção no processo semiautomático GMAW, apresentando dados sobre a natureza e magnitude das tensões residuais superficiais resultantes. Complementarmente é apresentada uma análise microestrutural para caracterização das juntas soldadas.

\section{MATERIAIS E MÉTODOS}

\subsection{MATERIAL}

O material utilizado neste trabalho foi o aço DP600 em forma de chapa de 4,15 mm de espessura. A composição química do material (fornecida pelo fabricante) está indicada na Tabela 1 e as propriedades mecânicas (estabelecidas pela norma SAE J2745) [5] são apresentadas na Tabela 2.

Tabela 1: Composição química do aço AHSS DP600 (em \% de peso).

\begin{tabular}{|c|c|c|c|c|c|c|c|c|c|}
\hline $\mathbf{C}$ & $\mathbf{S i}$ & $\mathbf{M n}$ & $\mathbf{P}$ & $\mathbf{S}$ & $\mathbf{A l}$ & $\mathbf{C u}$ & $\mathbf{C r}$ & $\mathbf{N i}$ & $\mathbf{F e}$ \\
\hline 0,04895 & 1,03 & 1,17 & 0,015 & 0,001 & 0,04 & 0,01 & 0,07 & 0,02 & Balanço \\
\hline
\end{tabular}


Tabela 2: Propriedades mecânicas do aço DP600 (norma SAE J2745).

\begin{tabular}{|c|c|c|}
\hline & Limite de Escoamento $\sigma_{\text {L.E. }}(\mathrm{MPa})$ & Limite de Resistência $\sigma_{\text {L.R. }}(\mathrm{MPa})$ \\
\hline Norma SAE J2745 & $340-440$ & $>600$ \\
\hline
\end{tabular}

\subsection{MÉTODOS}

\subsubsection{Soldagem GMAW}

As soldagens das amostras foram realizadas pelo processo GMAW, utilizando chanfro em $\mathrm{V}$ com $30^{\circ}$ de ângulo de bisel. Foram usadas duas misturas de gases de proteção: $92 \%$ de $\mathrm{Ar}+8 \% \mathrm{CO}_{2}$ (condição 1) e $75 \% \mathrm{Ar}$ $+25 \% \mathrm{CO}_{2}$ (condição 2), sendo os demais parâmetros mantidos para ambas as condições, conforme apresentado na Tabela 3. O eletrodo utilizado foi o ER70S-6 [14] com velocidade de alimentação de 6,4 mm/s. A composição química do eletrodo, conforme a norma AWS A5.18, está representada na Tabela 4.

Tabela 3: Parâmetros de soldagem utilizados no processo GMAW.

\begin{tabular}{c|c}
\hline Tensão (V) & 18 \\
\hline Corrente (A) & 140 \\
\hline Velocidade de soldagem $(\mathrm{mm} / \mathbf{s})$ & 6,4 \\
\hline Vazão do gás de proteção $(\mathrm{I} / \mathrm{min})$ & 12 \\
\hline
\end{tabular}

Tabela 4: Percentual de composição química do eletrodo ER70S-6 (em \% de peso) [14].

\begin{tabular}{c|c|c|c|c|c}
\hline $\mathbf{C}$ & $\mathbf{S i}$ & $\mathbf{M n}$ & $\mathbf{S}$ & $\mathbf{P}$ & $\mathbf{C u}$ \\
\hline $0,06-0,015$ & $0,80-1,15$ & $1,4-1,85$ & 0,035 máx & 0,025 máx & 0,5 máx \\
\hline
\end{tabular}

O processo de soldagem semiautomático foi realizado em uma máquina Fronius, modelo TransSteel $5000 \mathrm{MV}$,cabeçote VR 5000 4R/W/E Syn, com corrente contínua e polaridade reversa $\left(\mathrm{CC}^{+}\right)$. Ao total foram obtidas 3 amostras de cada condição, com dimensões de 40 x $510 \mathrm{~mm}$.

\subsubsection{Análise de tensões residuais}

As tensões residuais foram medidas e analisadas na superfície da junta, utilizando a técnica não-destrutiva de tensometria por difração de raios-X, pelo método $\operatorname{sen}^{2} \psi$, através de um analisador de tensões Stresstech, modelo XStress 3000, sob os parâmetros descritos na Tabela 5. As amostras foram medidas em ambos os lados da chapa (topo e raiz), nas direções transversal (T) e longitudinal (L) ao cordão de solda, na zona termicamente afetada (ZTA), metal de solda (MS) e metal de base (MB), conforme Figura 1. Para cada condição foi levantado um perfil de tensões residuais na direção transversal ao cordão de solda.

A análise microestrutural foi realizada por microscopia ótica, utilizando ataque Nital $2 \%$.

Tabela 5: Parâmetros para medição das tensões residuais.

\begin{tabular}{c|c}
\hline Diâmetro do colimador & $2,0 \mathrm{~mm}$ \\
\hline Ângulo $\mathbf{2 \theta}$ & $156,41^{\circ}$ \\
\hline Ângulos $\boldsymbol{\Psi}$ & $0 ; 20 ; 30 ; 40$ e $45^{\circ}$ \\
\hline Tempo de exposição & $10 \mathrm{~s}$ \\
\hline Corrente & $6,7 \mathrm{~mA}$ \\
\hline Tensão & $30 \mathrm{~V}$ \\
\hline
\end{tabular}



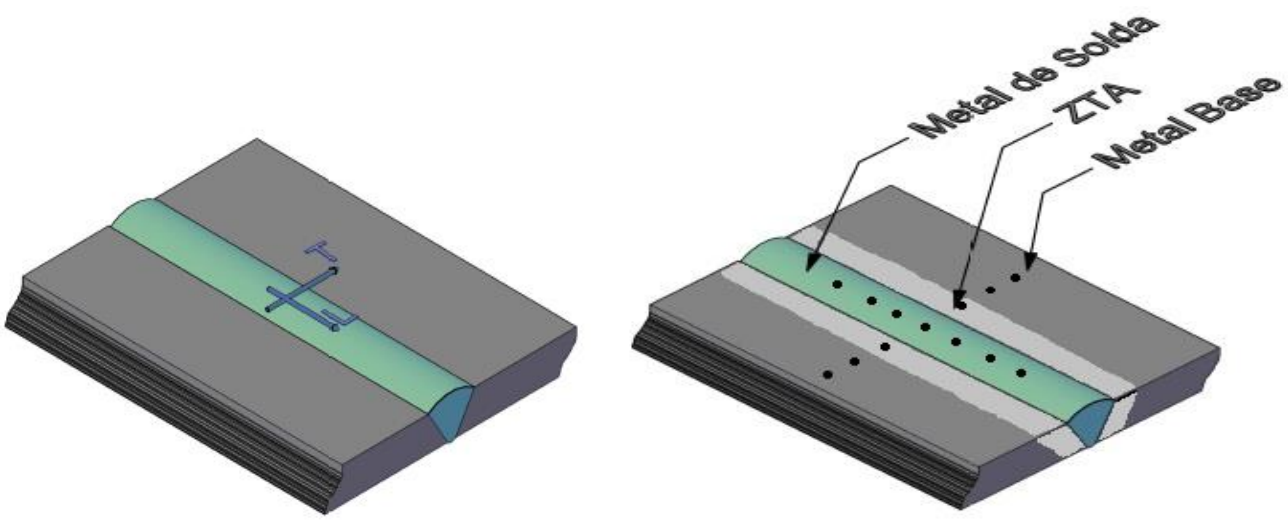

Figura1: Posições de medidas das tensões residuais nas diferentes regiões das juntas: T - transversal e L - longitudinal.

\section{RESULTADOS}

Antes da soldagem, foram medidas as tensões residuais (TR) do metal de base, cujos valores encontrados foram compressivos, com média de -145 MPa na direção transversal da chapa (sentido de laminação da chapa) e de -140 MPa na direção longitudinal, com erro de aproximadamente $15 \mathrm{MPa}$. A natureza compressivadas tensões residuais no metal de base é esperada, uma vez que as chapas utilizadas foram fabricadas por laminação. De maneira geral, os processos de conformação mecânica tendem a gerar um estado de tensões compressivo na superfície das peças em decorrência da deformação plástica inerente ao processo.

Analisando o gráfico da Figura 2, que mostra os valores médios das tensões residuais obtidos na ZTA na direção transversal, observa-se que as tensões residuais na raiz foram trativas em ambas as condições, com valores médios de maior magnitude para a condição com maior percentual de $\mathrm{CO}_{2}$ (condição 2). Já no topo as tensões apresentaram uma variação quanto à condição de soldagem. Na primeira condição (92\% de Ar $+8 \%$ $\left.\mathrm{CO}_{2}\right)$, as tensões foram trativas com uma média de $80 \mathrm{MPa}$ e na segunda condição $\left(75 \% \mathrm{Ar}+25 \% \mathrm{CO}_{2}\right)$ foram compressivas com uma média de $-120 \mathrm{MPa}$. Conforme apresentado por HEINZEN et al. [7], o ciclo térmico de soldagem influencia na geração das tensões residuais devido ao aquecimento em uma região localizada, com mudanças de fase, além de um resfriamento não uniforme da peça, acompanhado de gradientes térmicos que surgem entre a superfície e o núcleo da junta. Durante a soldagem é gerada condição de expansão e contração da zona fundida que tendem resultar em tensões residuais trativas da ZTA na direção transversal (tensões de contração). Por outro lado, algumas transformações de fase e o fator de resfriamento diferenciado entre a superfície e o núcleo da chapa propiciam a geração de tensões do tipo compressiva na ZTA. $\mathrm{O}$ acréscimo de $\mathrm{CO}_{2}$ na composição do gás de proteção leva à uma condição de maior penetração, que durante o ciclo de soldagem pode resultar em um campo de tensões compressivas na ZTA.

Na direção longitudinal (Figura 3) foi observado o mesmo padrão de comportamento da direção transversal, sendo a magnitude das tensões residuais da condição com menor percentual de $\mathrm{CO}_{2}$ maiores do que as medidas na direção transversal, para a mesma condição. Os valores altos de TR indicam que a contração de resfriamento foi muito mais severa na direção longitudinal do que na transversal ao cordão de solda. É importante destacar que tensões residuais trativas são deletérias pois podem propiciar uma menor vida em serviço dos componentes. Por outro lado, tensões residuais de natureza compressivas são benéficas, principalmente quando os componentes soldados são submetidos a esforços cíclicos, pois evitam a nucleação e propagação de trincas. 


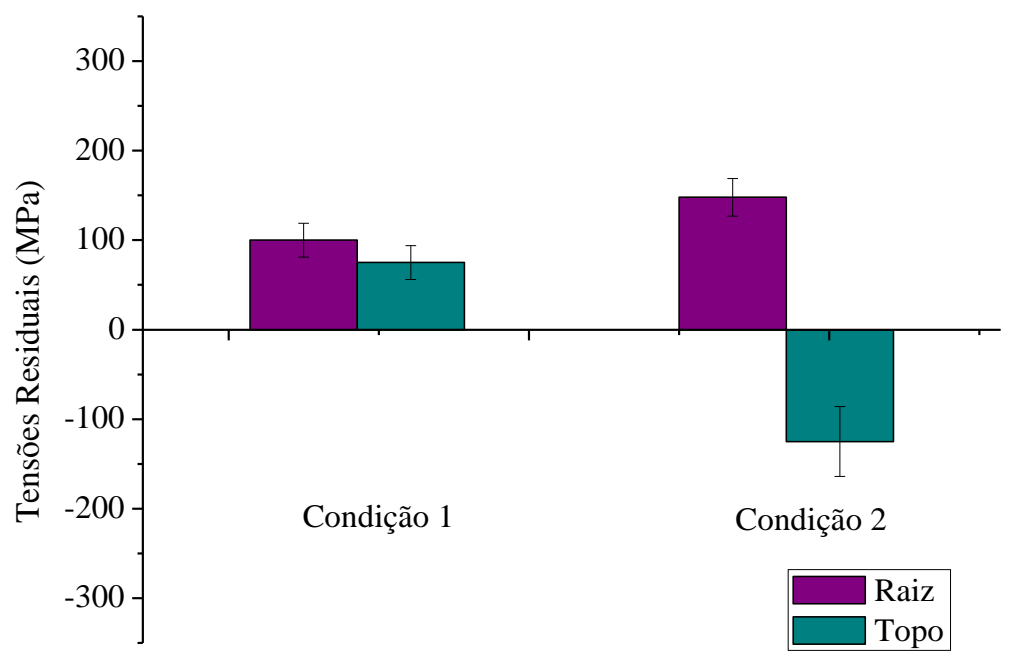

Figura 2: Tensões residuais na ZTA - transversal.

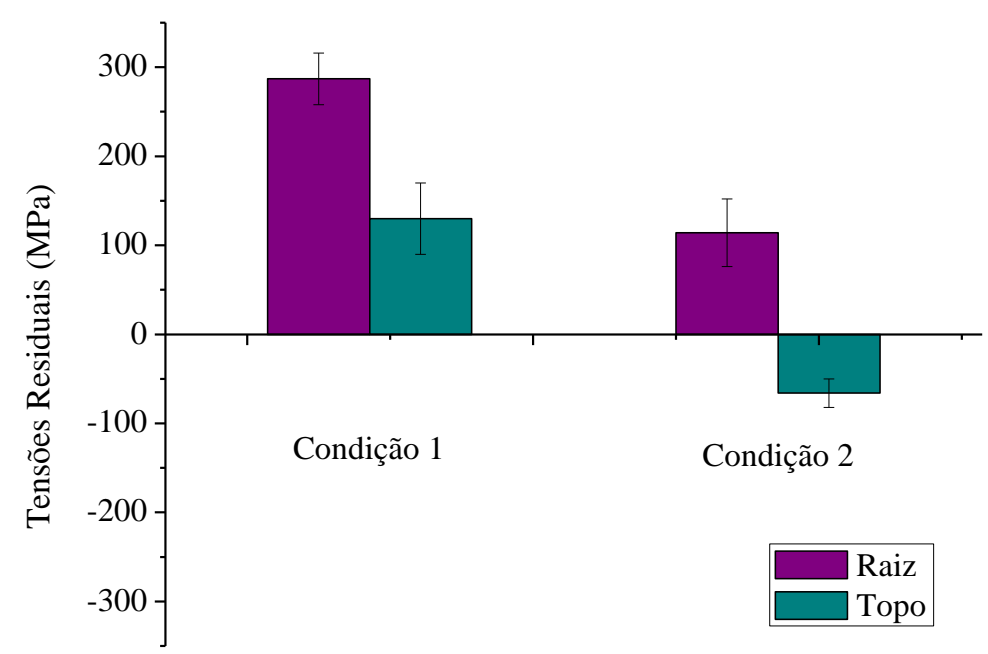

Figura 3: Tensões residuais na ZTA - longitudinal.

Nas Figuras 4 e 5 são apresentadas as médias dos valores de tensão residual na região do metal de solda. Analisando a Figura 4, nota-se uma característica compressiva em ambas as condições de soldagem para a raiz, com um valor médio maior na condição 2. A natureza compressiva da região do metal de solda, na raiz das juntas soldadas, pode estar ligada a geometria da junta soldada (Figura 8), onde se observa um acúmulo de metal de solda em uma região restrita. Durante o resfriamento da junta soldada, a região central da mesma tende a ficar em compressão devido à contração volumétrica, conforme os resultaram mostraram. No topo das juntas, as tensões apresentaram característica trativa na condição 1, com uma média de $200 \mathrm{MPa}$, enquanto que na condição 2 pode ser observado uma característica compressiva, com média de -100 MPa. Os padrões de TR estão relacionados diretamente com a expansão e contração de resfriamento, além da carga térmica modificada pelo aumento do percentual de $\mathrm{CO}_{2}$ na mistura do gás de proteção. $\mathrm{O}$ aumento do teor de $\mathrm{CO}_{2}$ gera um arco mais agressivo e com maior condutividade térmica, resultando em um gradiente térmico de maior intensidade na direção transversal ao cordão de solda, levando a um estado compressivo no centro do mesmo. Ressalta-se ainda que o aumento do percentual de $\mathrm{CO}_{2}$ pode alterar o modo de transferência metálica, que pode vir a influenciar diretamente no estado das tensões residuais na junta soldada.

Na direção longitudinal do metal de solda (Figura 5), nota-se que as tensões tiveram mesmo compor- 
tamento apresentado na direção longitudinal da ZTA, alterando apenas a magnitude dos valores medidos, comportamento esperado em virtude do menor volume de material nesta direção, o que facilita o escoamento uniforme da junta.

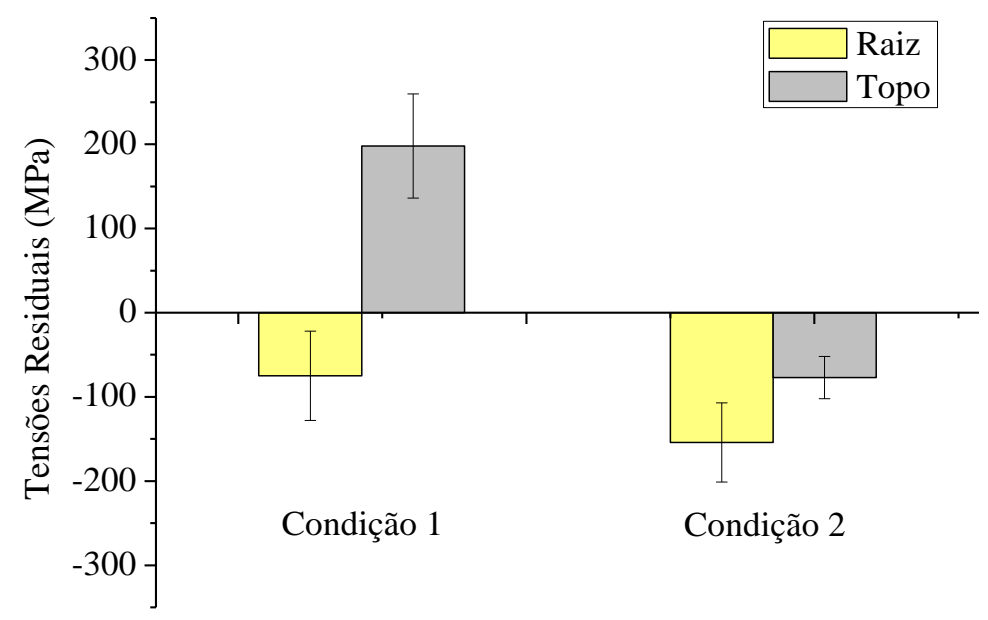

Figura 4: Tensões residuais no metal de solda - transversal.

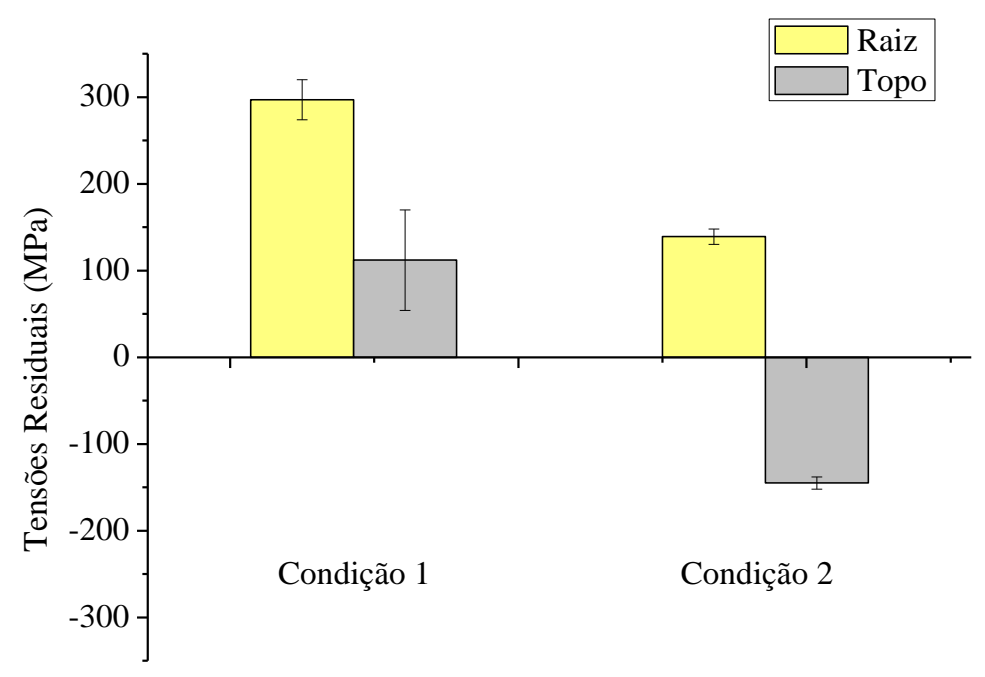

Figura 5: Tensões residuais no metal de solda - longitudinal.

Com o intuito de detalhar o comportamento das tensões residuais, foram obtidos perfis para cada condição na direção transversal ao cordão de solda. A Figura 6 apresenta o perfil dos valores das tensões da raiz de ambas as condições de soldagem, onde o metal de solda tem um estado de tensões compressivo e as ZTAs estão em tração. Entretanto, na Figura 7, que representa o perfil dos valores das tensões residuais no topo, pode-se observar o diferente comportamento na região central do cordão de solda. Para a condição 1 , com menor concentração de $\mathrm{CO}_{2}$, observa-se um caráter trativo no metal de solda, que pode ser resultante resfriamento mais intenso do que o apresentado na condição 2 , de maior concentração de $\mathrm{CO}_{2}$. 


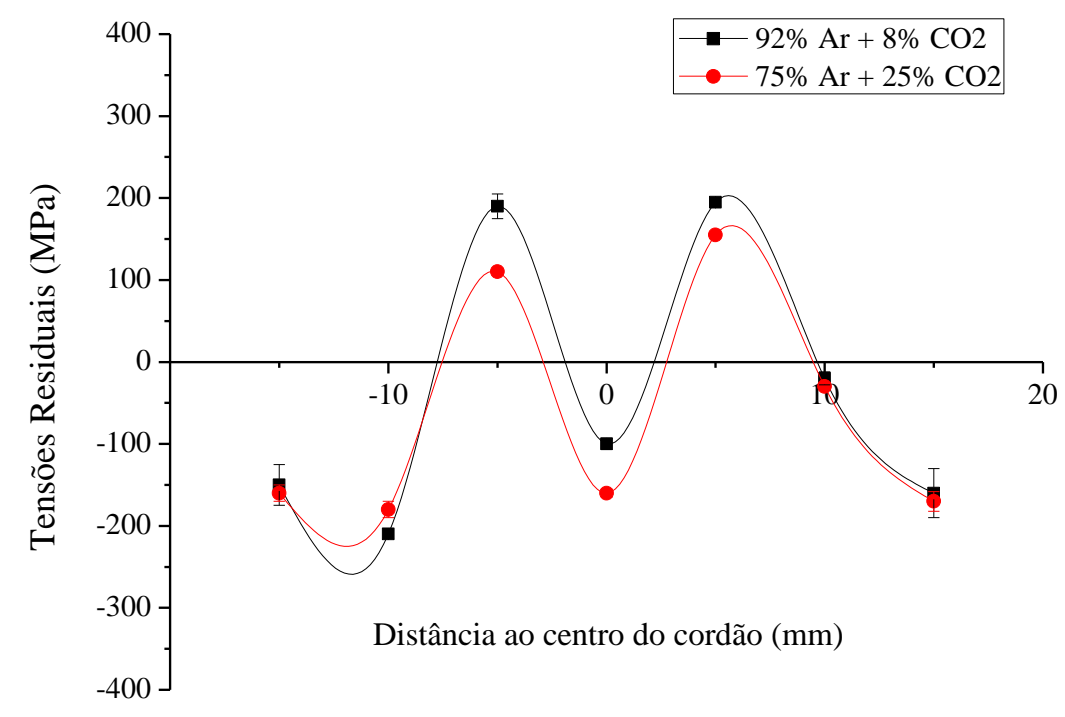

Figura 6: Perfil transversal das amostras - raiz.

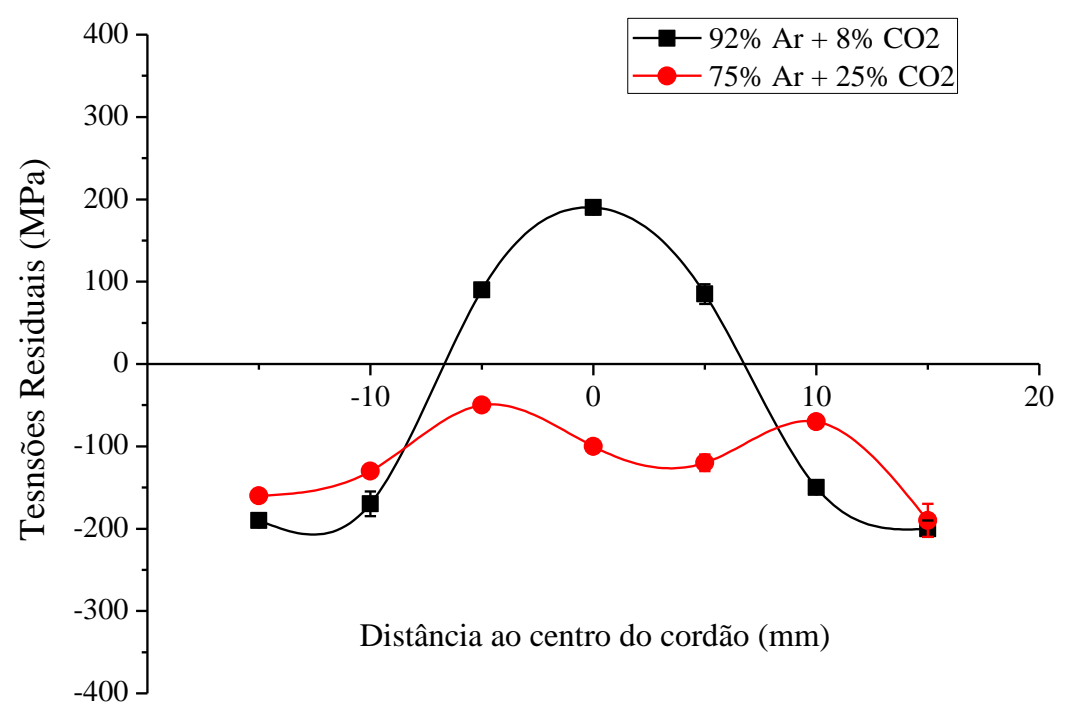

Figura 7: Perfil transversal das amostras - topo.

A microestrutura das juntas soldadas pode ser observada na Figura 8, onde verifica-se uma microestrutura menos grosseira na primeira condição $\left(92 \%\right.$ de $\left.\mathrm{Ar}+8 \% \mathrm{CO}_{2}\right)$, com presença de martensita, decorrente do rápido resfriamento da poça de fusão durante o processo de soldagem, e matriz ferrítica, similares as microestruturas encontradas por FARABI et al. [3] e FARABI et al. [4]. 


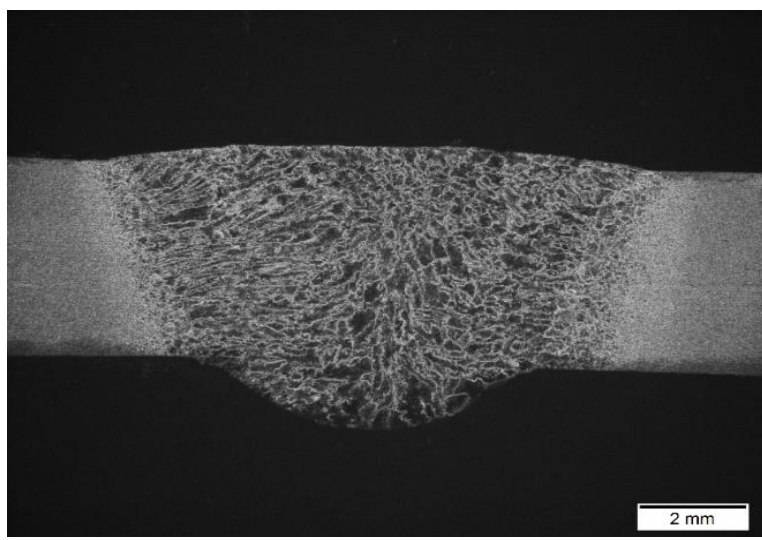

(a)

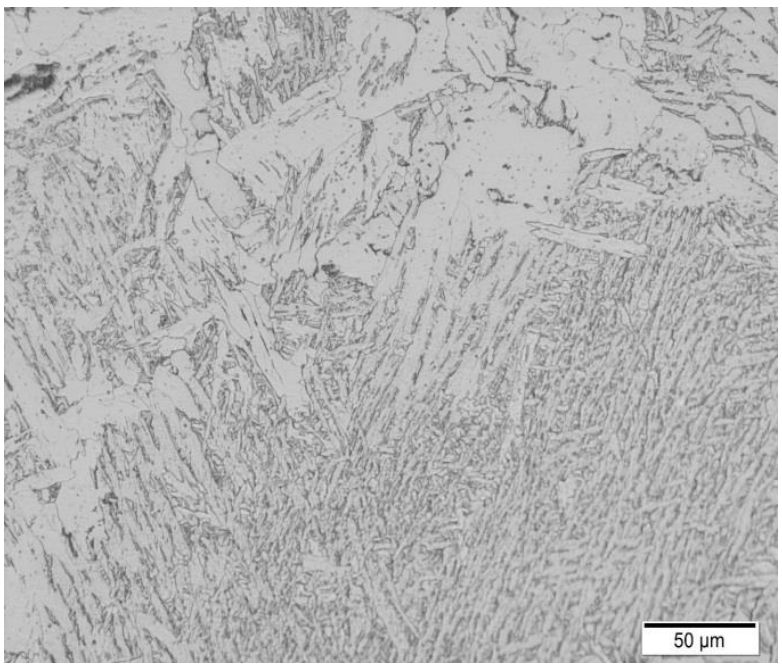

(c)

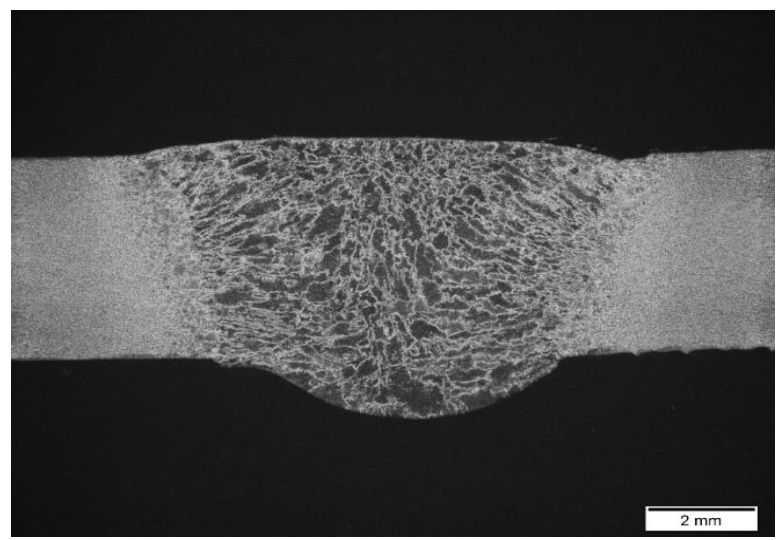

(b)

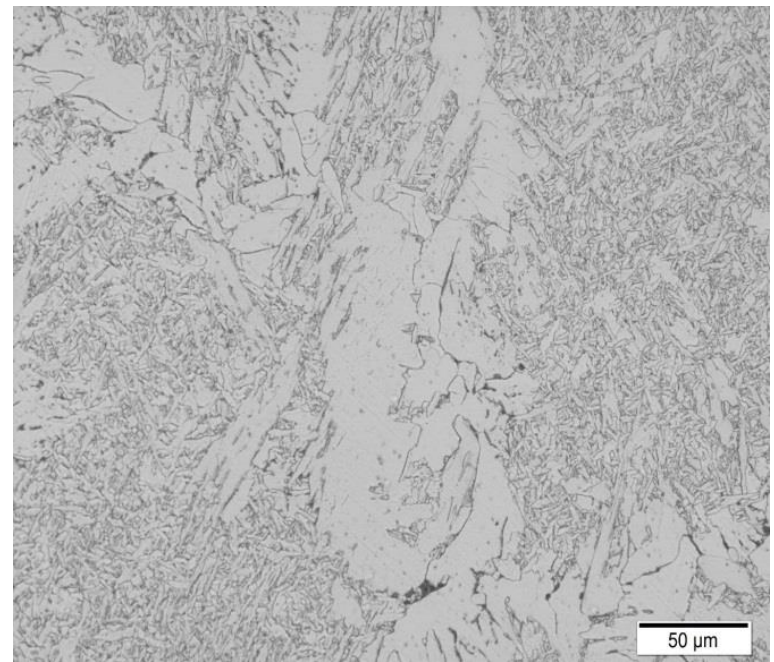

(d)

Figura 8: Macrografia das juntas soldadas: (a) condição 1 (92\% de $\mathrm{Ar}+8 \% \mathrm{CO} 2)$ e (b) condição $2(75 \% \mathrm{Ar}+25 \%$ CO2). Micrografia do metal de solda: (c) condição 1 e (d) condição 2.

Nota-se na Figura 8b que a geometria do cordão de solda da amostra soldada na condição 2 é mais estreita do que a amostra soldada na condição 1, característica diretamente ligada a quantidade de $\mathrm{CO}_{2}$ introduzida na mistura. Conforme a literatura [10] obtém-se maiores penetrações com cordões mais estreitos quando se aumenta a quantidade de $\mathrm{CO}_{2}$ na mistura $\mathrm{Ar}-\mathrm{CO}_{2}$. O aumento do percentual de $\mathrm{CO}_{2}$ tende a gerar um arco mais severo, elevando os níveis de respingos, a convexidade do cordão e a penetração da solda. A alta condutividade térmica do $\mathrm{CO}_{2}$ é responsável por uma alta transferência de calor para a poça de fusão, que contribui diretamente para geração das tensões residuais.

\section{CONCLUSÕES}

O presente trabalho, que teve por objetivo analisar as tensões residuais provenientes da soldagem GMAW de um aço DP600, permite as seguintes conclusões:

1. As tensões residuais na ZTA apresentam natureza trativa nas direções transversal e longitudinal ao cordão de solda, tanto no topo quanto na raiz, na condição com $92 \%$ de $\mathrm{Ar}+8 \% \mathrm{CO}_{2}$. Para a condição com $75 \% \mathrm{Ar}+25 \% \mathrm{CO}_{2}$, a raiz também apresenta valores trativos, entretanto no topo das juntas foram observados valores de tensões residuais em compressão.

2. Na região do metal de solda foi observado que aumentado o percentual de $\mathrm{CO}_{2}$ na mistura do gás houve uma tendência de geração de tensões compressivas, tanto na raiz quanto no topo das juntas, no sentido transversal a junta. 
3. Analisando os dados de forma mais abrangente, pode-se notar que a condição $75 \% \mathrm{Ar}+25 \% \mathrm{CO}_{2}$, onde se têm maior quantidade de $\mathrm{CO}_{2}$, apresentou uma tendência compressiva o que seria benéfico à junta com relação às tensões residuais, enquanto que a condição com $92 \%$ de $\mathrm{Ar}+8 \% \mathrm{CO}_{2}$ apresenta um padrão trativo, que poderia ser prejudicial. Portanto, nesse estudo fica evidenciado que a composição do gás de proteção é um parâmetro que influencia a natureza e magnitude das tensões.

4. A microestrutura encontrada das juntas para as duas condições estudadas foi basicamente martensítica e matriz ferrítica, conforme esperado.

\section{AGRADECIMENTOS}

Os autores agradecem à CAPES, ao CNPQ e à FAPERJ pelo auxílio financeiro que possibilitou a realização desse trabalho.

\section{BIBLIOGRAFIA}

[1] SILVA FILHO, J.F., OLIVEIRA, C.A.S., FONSTEIN, N., et al., "Effect of Cr additions on ferrite recrystallization and austenite formation in dual-phase steels heat treated in the intercritical temperature range", Materials Research, v. 19, n. 1, pp. 258-266, 2016.

[2] CINGARA, G., OSOSKOV, Y., JAIN, M.K., et al., "Effect of martensite distribution on damage behavior in DP600 dual phase steels", Materials Science and Engineering A, v.516, n. 1-2, pp. 7-16, 2009.

[3] FARABI, N., CHEN, D.L., ZHOU, Y. "Microstructure and mechanical properties of laser welded DP600 steel joints", Journal of Materials Science and Engineering, v.527, pp. 1215-1222, 2011.

[4] FARABI, N., CHEN, D.L., ZHOU, Y. "Microstructure and mechanical properties of laser welded dissimilar DP600/DP980 dual-phase steel joints", Journal of Alloys and Compounds, v.509, pp. 982-989, 2010.

[5] SOCIETY OF AUTOMATIVE ENGINEERS. J2745: Surface vehicle recommended practice: Categorization and Properties of Advanced High Strength Automotive Sheet Steels, 2007.

[6] WHITERS, P.J., BHADESHIA, H.K.D.H. "Residual stress: Part 1 - Measurement techniques", Materials Science and Technology, v. 17, pp. 355-365, 2001.

[7] HEINZE, C., SCHWENK, C., RETHMEIER, M., "Numerical calculation of residual stress development of multi-pass gas metal arc welding”, Journal of Constructional Steel Research, v. 72, pp. 12-19, 2012.

[8] RODRIGUES, L.D., FREIRE, J.L., VIEIRA, R.D. "Desenvolvimento e avaliação experimental de uma nova técnica para medição de tensões residuais”, Matéria, v.16, n. 4, pp. 842-856, 2011.

[9] DIAS, J.S., CHUVAS, T.C., CINDRA FONSECA, M.P. "Evaluation of Residual Stresses and Mechanical Properties of IF Steel Welded Joints by Laser and Plasma Processes", Materials Research, v. 19, p. 721$727,2016$.

[10] MUKHOPADHYAY, S., PAL, T.K. "Effect of shielding gas mixture on gas metal arc welding of HSLA steel using solid and flux-cored wires", International Journal of Advanced Manufacturing Technology, v.29, pp. 262-268, 2006.

[11] RAO, Z.H., HU, J., LIAO, S.M., et al., "Modeling of the transport phenomena in GMAW using argonhelium mixtures. Part I - The arc", International Journal of Heat and Mass Transfer, v. 53, pp. 5707-5721, 2010.

[12] DUTRA, J.C. "MIG/MAG - Transferência Metálica por Curto-Circuito - Fontes de Soldagem versus Gases do Arco", Soldagem Inspeção, São Paulo, vol. 13, n. 1, pp. 019-024, 2008.

[13] WANG, L.L., LU, F.G., WANG, H.P., et al., "Effects of shielding gas composition on arc profile and molten pool dynamics in gas metal arc welding of steels", Journal of Physics D: Applied Physics, v.47, n. 46, pp. $465202+14,2014$.

[14] BELGO BEKAERT ARAMES. http://belgobekaert.com.br/Produtos/Documents/Folder-Solda.pdf. Acessado em: 01. jun. 2017. 


\section{ORCID}

Thiago Menezes Maia

Hector Reynaldo Meneses Costa

Maria Cindra Fonseca

Tatiane de Campos Chuvas https://orcid.org/0000-0002-2048-9769

https://orcid.org/0000-0002-0887-6838

https://orcid.org/0000-0001-9584-8394

https://orcid.org/0000-0002-7512-4193 